xii

$\therefore \quad \lambda_{1} \lambda_{2} \lambda_{3}=\left|\begin{array}{lll}a, & h, & g \\ h, & b, & f \\ g, & f, & c\end{array}\right|$.

Using the values of $a, b, \ldots$ above, it is easy to show that

$$
\frac{a l_{1}+h m_{1}+g n_{1}}{l_{1}}=\frac{h l_{1}+b m_{1}+f n_{1}}{m_{1}}=\frac{g l_{1}+f m_{1}+c n_{1}}{n_{1}}=\lambda_{1}
$$

and so to obtain the result, but the direct method is of some interest.

R. J. T. BELL.

\title{
A Geometrical Proof for Hero's Formula.
}

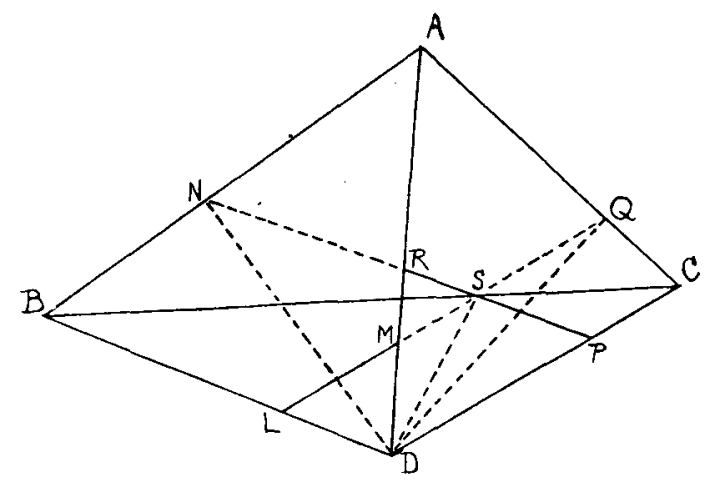

The following proof is designed to link up" Hero's formula geometrically with the formulae for the trigonometrical functions of $\frac{1}{2} \mathrm{~A}$ in a triangle.

From the bisector of angle $A$ let $A D$ be cut off equal to the mean proportional between $A B$ and $A C$, and let $N$ be the projection of $D$ on $A B$. The formulae $A N=\sqrt{s(s-a)}, N D=\sqrt{(s-b)(s-c)}$ are easily established geometrically, and are assumed here. Thus triangle $A N D$ gives directly the formulae for $\sin \frac{1}{2} A, \cos \frac{1}{2} A, \tan \frac{1}{2} A$. Hero's formula is thus represented by $A N . N D$. It is required to prove therefore that twice the area of triangle $A N D$ is equal to the area of triangle $A B C$.

Join $B D, D C$ and draw the pedal triangles $L M N, P Q R$ of the triangles $A B D, A D C$, which are similar since $A B: A D=A D: A C$. These triangles are then divided by their pedal triangles into similar component pairs, and the three triangles round a pedal triangle 
are all similar to the whole triangle. Now $P R$ and $Q R$ are equally inclined to $A D$ (a pedal property), and $N$ is the image of $Q$ in $A D$. $\therefore P R$ produced passes through $N$; similarly $L M$ produced passes through $Q$. Let these lines cut $B C$ in $S$ and $S^{\prime}$.

Again $P N \| D B$ since alternate angles $B D R, D R P$ are corresponding angles of the similar triangles $B D A, D R P$; similarly $L Q \| D C$.

$$
\text { But } \begin{aligned}
B S: S C & =D P: P C & & (B D \text { parallel to } S P) \\
& =B L: L D & & \text { (complete similarity of the figures) } \\
& =B S^{\prime}: S^{\prime} C & & \left(L S^{\prime} \text { parallel to } D C\right)
\end{aligned}
$$

$\therefore S$ and $S^{\prime}$ coincide.

Now area of triangle $N B S=$ area of triangle $N D S$ ( $N S$ parallel to $B D$ )

$$
\text { , } \quad Q C S=\quad, \quad, \quad Q D S(Q S \quad, \quad D C) .
$$

To the sum of these areas add area $A N S Q$.

$\therefore$ in area, triangle $A B C=$ kite $A N D Q=$ twice triangle $A N D$.

G. D. C. STokes.

Editor's Notw.-Mr John T. Brown suggests the following neat method of proving that the triangle $A B C$ is twice the triangle $A N D$ :

Suppose $D N$ produced its own length to $E$.

Then the angles $E A D, B A C$ are equal,

$$
\text { and } A E \cdot A D=A J^{2}=A B \cdot A C \text {. }
$$

Hence, by Euc. VI, 15, the triangles $A E D, A B C$ are equal; i.e. twice triangle $A N D=$ triangle $A B C$.

$$
\text { W. A. }
$$

\section{A Proof of the Theorem of Pythagoras.}

The triangle $A B C$ has a right angle at $B$. On $A C$, on the same side as $B$, describe a square $A D E C$. Draw $D F$ perpendicular to $A B$ or $A B$ produced.

The triangles $A B C$ and $D F A$ are congruent, having sides $C A$ and $A D$ equal, and the corresponding angles equal. Hence $D F$ is equal to $A B$. 